\title{
Outcomes of Ruptured Sinus of Valsalva Aneurysm -A 20-year experience
}

\author{
Harshavardhan Niraghatam ${ }^{1}$, Dipika Naraimathi ${ }^{1}$, Utkarsh Sanghavi ${ }^{1}$, Aditya N. \\ Doddamane $^{1}$, Channabasavaraj Hiremath ${ }^{2}$, and Shekhar Rao ${ }^{1}$ \\ ${ }^{1}$ Sri Sathya Sai Institute of Higher Medical Sciences Whitefields \\ ${ }^{2}$ Affiliation not available
}

April 30, 2021

\begin{abstract}
Background: Ruptured aneurysms of sinus of Valsalva are defects of the aortic media, which are uncommon yet present concomitantly with either ventricular septal defect, aortic insufficiency or both. Here, we analyse retrospectively outcomes of patients operated on in the last two decades. Patients and Methods: 151 cases of ruptured aneurysms of sinuses of Valsalva were treated here between January 2000 and December 2020. The majority (96\%) arose from the right coronary sinus. The right ventricular outflow was the most common site of rupture (56.2\%). Ventricular septal defect was associated in 24 patients $(16 \%)$ all of whom had ruptured right sinus, of which $8(33 \%)$ were of subpulmonic subtype. Aortic incompetence was found in 45 patients $(43.3 \%)$. Elective surgery was offered to 78 patients $(51.6 \%)$, while the rest had defects closed by interventional devices. Surgical conversion for device embolization occurred in 12 patients $(7.94 \%)$. The defect was closed through the aortic root in 13 patients (14.4\% of 90) and employing the bicameral technique (root and ruptured chamber) in the remaining 87 patients. 18 patients $(20 \%)$ underwent aortic valve repair while $5(5.5 \%)$ underwent aortic valve replacement. Results: We had no hospital deaths, however 4 operated patients (2.6\%) had comorbidities. Follow-up ranged from 1 to 20 years (mean $13 \pm 5$ ). There were 3 deaths in this period, and among the majority, quality of life was uneventful. Conclusion: Surgical correction for ruptured aneurysms of Valsalva provides prudent results and must be the preferred modality of treatment in acute and chronic presentation.
\end{abstract}

Outcomes of Ruptured Sinus of Valsalva Aneurysm -A 20-year experience. TITLE PAGE

\section{Authors List}

1. Niraghatam Harshavardhan MS, MCh

2. Dipika Naraimathi MBBS, (DNB)

3. Utkarsh Sanjay Sanghavi MBBS, (DNB)

4. Aditya Narsipur Doddamane BTech, (MD)

5. Channabasavaraj Shivalingaiah Hiremath MNAMS, MCh

6. Shekhar Rao MS, MCh

\section{Affiliation}

Division of Cardiothoracic \& Vascular Surgery

Sri Sathya Sai Institute of Higher Medical Sciences

EPIP Area, Whitefield, Bengaluru - 560066, INDIA 


\section{Corresponding Author Details}

Channabasavaraj Shivalingaiah Hiremath MNAMS, MCh

Professor \& Head of Department

Division of Cardiothoracic \& Vascular Surgery

Sri Sathya Sai Institute of Higher Medical Sciences

EPIP Area, Whitefield, Bengaluru - 560066, INDIA

Email: hiremath.cs@sssihms.org.in

Phone: +91 9481119696/ 7975418597

\section{Data Availability Statement}

Data available on request from the authors

\section{Declarations}

Funding: None

Conflict of interest: None

Outcomes of Ruptured Sinus of Valsalva Aneurysm -A 20-year experience.

Abbreviations:

RSOV- Ruptured Sinus of Valsalva

NYHA- New York Heart Association

VSD- Ventricular Septal Defect

AR- Aortic Regurgitation

RVOT- Right ventricular outflow tract

CTR-Cardio-Thoracic Ratio

CXR- Chest Roentgenogram

ECG- Electrocardiogram

RBBB- Right Bundle Branch Block

LBBB- Left Bundle Branch Block

CHB- Complete Heart Block

INR- International Normalized Ratio

e-PTFE- expanded Poly Tetra Flouro Ethylene

SAM- Sub Aortic Membrane

OS-ASD- Ostium Secundum Atrial Septal Defect

PDA- Patent Ductus Arteriosus

2D Echo- 2Dimensional Echocardiography

Introduction:

Sinus of Valsalva aneurysm is a rare cardiac anomaly, with its etiology being congenital or acquired. It was first described by John Thurman in 1840 . Incidence is 0.14 to $3.5 \%$ in patients undergoing open heart surgical 
procedures [1], and comprises up to $3.5 \%$ of all congenital cardiac anomalies with a higher incidence in Asian $(1.2 \%$ to $4.94 \%)$ than in Western population $(0.5 \%-1.5 \%)$ [2]. RSOV can be congenital or acquired. Congenital RSOV is due to weakness at the junction between the aortic media and the annulus fibrosus, whereas acquired RSOV is secondary to infective endocarditis, syphilitic aortitis, atherosclerosis or connective tissue disease [3,4]. Type I RSOV as per modified Sakakibara classification is the most common morphological type [5]. The associated cardiac anomalies both VSD and aortic valve incompetence should always be carefully looked for [4]. If RSOV is not treated, it may lead to myocardial infarction from compression of coronary artery, complete heart block from compression of conduction tissues, RVOT obstruction, infective endocarditis, cardiac tamponade or may be a source of cerebrovascular emboli. Once rupture has occurred, mean survival is 1 to 2 years. Death is usually due to congestive heart failure, but infective endocarditis has been the cause of death in approximately $8 \%$ of cases [6]. Herein, we assess the postoperative outcomes of surgical repair and device closure in the management of ruptured aneurysms of sinus of Valsalva cases at our institution over a period of 20 years from 2001 till 2020.

\section{Materials and methods:}

This is a Retrospective case series where we have referred the medical records of the patients over the last 20 years after due permission from the Institutional Review Board and Informed Patient Consent obtained from all operated patients as a matter of hospital policy and good clinical practice. From January 2001 till December 2020, a total of 151 patients were diagnosed to have ruptured Sinus of Valsalva aneurysm at our institute. 139 patients were reachable in the follow up interview over the telephone to ascertain their alive and functional status.

All patients were evaluated by the cardiologist by a 2D-Echocardiography (2D Echo) and cardiac catheterization whenever indicated and those amenable to device closure were taken up for device closure. Candidates who had the complication of device embolization post device closure and those with other associated lesions such as large juxta arterial and peri membranous Ventricular Septal Defects (VSD), moderate to severe Aortic Regurgitation (AR), Mitral Regurgitation (MR), Tricuspid Regurgitation (TR), Ostium Secundum -Atrial Septal Defect (OS-ASD) not possible for device closure were referred for surgical management. Device closure was performed using devices such as Cocoon VSD Occluder device (Vascular innovations, Nathambury, Thailand), Amplatzer Duct Occluder (AGA Medical Corporation, Golden Valley, MN). Associated muscular VSD was closed using Amplatzer muscular VSD Occluder (AGA Medical Corporation, Golden Valley, MN). Surgical repair was performed using cardiopulmonary bypass under moderate hypothermia. For RSOV repair and VSD closure various patch materials such as autologous treated pericardial patch with $0.6 \%$ Glutaraldehyde, Dacron and e-PTFE. Intraoperatively, the presence or absence of associated VSD was always looked for carefully irrespective of 2D-Echo and Cardiac Catheterization findings.

The statistical analysis was performed using RStudio (Vienna, version 4.2, 2020) software. A 20year survival analysis was performed using Kaplan Meier regression model and with hazard ratio analysis. Categorical variables were analyzed using Chi square test and numerical data was analyzed using Student's t-test and expressed as mean \pm standard deviation. Patients lost to follow-up were right-censored at the time of last follow-up to avoid bias.

\section{Results:}

We observed a female predominance by $29 \%$. Presenting symptoms were predominantly dyspnea ranging from NYHA I-NYHA IV, majority in NYHA class II (63\%) \& palpitations and other symptoms such as angina, fatigue \& heart failure. Continuous murmur with thrill was present all patients along the left parasternal border. Preoperatively patients presented with several rhythm abnormalities such as sinus tachycardia, atrial fibrillation, Right Bundle Branch (RBBB), Left Bundle Branch Block (LBBB) \& Complete Heart Block (CHB) Table 1 . Chest roentgenograms (CXR) showed cardiomegaly of varying degrees, and the mean cardiothoracic ratio (CTR) before operation was $58.3 \pm 5.6$. As per the modified Sakakibara classification [5], Type I RSOV was the most common morphology of RSOV identified with prevalence of $38.4 \%$. RSOV was associated with several intracardiac lesions most common being Ventricular Septal Defect (VSD) 15.89\%, 
other associated lesions are Tricuspid Regurgitation (TR), Sub Aortic Membrane (SAM), Aortic Regurgitation (AR), Mitral regurgitation (MR), pulmonary Regurgitation (PR), ostial secundum Atrial Septal Defect (OS-ASD) Table 2 .

A total of 61 uncomplicated device closures (40.39\%) have been performed. 12 cases (7.94\%) had complication of device embolization which were taken up for emergency device retrieval and RSOV repair. Elective surgical repair of RSOV along with repair of associated intra cardiac lesions was performed in 78 cases $(51.65 \%)$. Bicameral approach using Trans Aortic-Trans Pulmonary, Trans Aortic-Trans Right Atrium, Trans AorticTrans Left Atrium based on the type of associated lesions was most commonly used for repair of RSOV in 77 cases $(85.5 \%)$ both during elective and emergency surgeries. Autologous treated pericardium was the most commonly used patch material in 61 cases $(67.77 \%)$. Among the concomitant procedures, VSD patch closure and Tricuspid valve repair using modified DeVega's technique were the most commonly performed procedures in 22 cases $(24.44 \%)$. Aortic valve repair was performed using Trussler's leaflet repair technique in 18 cases $(20 \%)$ \& Valve replacement was performed using TTK Chitra Valve (TTK Healthcare Ltd, Chennai, India) in 5 cases (5.55\%), SAM excision was performed in 10 cases (11.11\%), Pulmonary valve repair \& Right Ventricular Outflow Tract (RVOT) muscle bundle resection in 1case each (1.11\%), Patent Ductus Arteriosus (PDA) ligation in 2 cases $(2.22 \%)$ and ASD closure in 3 cases (3.33\%) Table 3 .

During the postoperative period, 2 patients $(2.22 \%)$ who underwent surgical repair for RSOV had ischemic stroke of which 1patient underwent elective surgical RSOV repair and other patient underwent an emergency RSOV repair following device embolization. 2 patients $(2.22 \%)$ in the elective surgical repair arm developed Complete Heart Block (CHB) in the postoperative period and they were discharged after Permanent Pace maker Implantation (PPI). The mean postoperative hospital stay in the device group was significantly shorter compared to the elective surgical repair group $4 \pm 2$ days vs $9 \pm 4$ days $(\mathrm{P}<0.0001)$, but there was no statistically significant difference observed with respect to the postoperative hospital stay between elective surgical repair group vs emergency surgical repair group post device embolization $9 \pm 4$ vs $13 \pm 5$ days $(\mathrm{P}>0.05)$. On postoperative follow up 2D-Echocardiography, at 3months 1 patient $(1.63 \%)$ in the device closure arm had residual RSOV and was surgically repaired with bicameral approach. The device failure rate in this study is calculated to be $17.8 \% .3$ patients (3.84\%) in the elective surgical arm and 1patient (8.33\%) in the emergency surgical arm following device embolization continued to have severe left ventricular dysfunction on postoperative 3months and 12 months follow up 2D-Echocardiography similar to their preoperative. 1 patient in the emergency surgical arm post device embolization who underwent concomitant Aortic Valve repair was diagnosed to be having severe Aortic regurgitation on 3months follow up 2D-Echocardiography, he underwent Aortic Valve replacement with mechanical prosthetic TTK Chitra Valve (TTK Healthcare Ltd, Chennai, India) Table 4.

\section{Functional Class Assessment: Figure 1a, 1b}

In an assessment at one-year follow-up to determine whether patients were relieved of their presenting symptoms, a total of 4 patients reported no improvement in quality of life, thereby being interpreted as NYHA functional class status quo. Mean follow-up duration was $67 \pm 42$ months for the entire cohort of patients. Patients receiving surgical treatment were followed-up across a duration of $77 \pm 47$ months among whom 3 patients $(3.7 \%)$ reported no change in symptoms following surgery. Median duration for improvement in symptoms among the remaining patients (75) who received surgical treatment was 1.5 months (range 12 months) $(\mathrm{p}<0.001)$. Those patients who received percutaneous device closure were followed-up across a duration of $54 \pm 26$ months among whom no patients reported discomfort presenting as symptoms. The median duration for improvement was 1 month (range 0.6-1 month). Patients who had conversion from percutaneous device closure to open surgical repair were followed up for a duration of $71 \pm 55$ months over 20 years. 1 patient (8.3\%) was symptomatic even after treatment while the remaining subset of patients reported relief in symptoms at a median duration of 1.25 months (range 1-3.25 months). A Chi square coefficient of 27.3 for a Mantel Haenszel test-statistic of $\mathrm{P}<0.001$ shows a significant difference in symptom relief across the three groups.

Survival analysis: Figure 2a, 2b 
In our experience, there were no cases of in-hospital mortality ( $<30$ days) \& reoperation. All 7 mortalities were within the range of 6 months-11 years (median of 7 years). With the Kaplan-Meier regression model, we deduced an actuarial survival rate of $76.2 \%$ (C.I: $56.5-100$ ) at the end of the 20-year study period. In a mortality of seven patients, six patients who underwent corrective surgery succumbed to various causes including cerebrovascular accidents and sudden death within a mean duration of $84 \pm 41$ months following surgery. One patient who underwent percutaneous device closure succumbed at 6 months following procedure. Subgroup analysis of all three groups report an actuarial survival of $70.2 \%$ for the surgical group and $98.3 \%$ for the percutaneous device group over a 20year period. There were no early/ late mortalities in the conversion groups. Study of significance between the mortalities across treatment groups with Log-log rank/ Mantel-Haenszel algorithm brought forward a test-statistic of 0.5 , revealing no statistical difference across the treatment groups.

\section{Discussion:}

Direct comparison of outcomes of RSOV device closure and surgical repair would be confounding when not accounting complex concomitant lesions into perspective with recommended rules of practise in the present milieu. The largest series of RSOV available is published by Kuriakose EM et al. [6] which is a systematic review of 34 studies comprising of 877 patients over a span of 58 years from 1956-2014. Review of 16 studies comprising of 741 patients in the surgical repair cohort with a mean follow up of 9 years and 37 studies comprising 136 patients with a mean follow up of 5 years duration in the device closure cohort. Concomitant aortic valve repair was performed in $20 \%$ of surgical cohort and the incidence of new onset AR post aortic valve repair was found to be $1.8 \%$ and that of progressive $\mathrm{AR}$ was $6.8 \%$ which led to redo aortic valve repair surgery rate of $4.2 \%$. In the device closure group, conversion rate to open surgery of $2.9 \%$. In the device closure cohort, device failure rate is $2.9 \%$. Outcomes of our series are comparable with the above series as the concomitant aortic valve repair was performed in $20 \%$ of surgical repair group with an incidence of progressive AR of $5.55 \%$, which led to redo aortic valve surgery. The device failure rate in our study is $17.8 \%$. which is high. In the systematic review by Kuriakose EM et al. [6], there was a cumulative early (<30days) mortality of $1.5 \%$ and that of late mortality is $3.2 \%$ in the surgical cohort. In our series there is an actuarial survival of $70.2 \%$ for the surgical group and $98.3 \%$ for the percutaneous device group over a 20year period. Moustafa $\mathrm{S}$ et al. [7] in their study had a survival rate of $63 \%$ at the end of 10 years in the surgical cohort of RSOV repair. In an Indian study by Choudhary SK et al. [8] of 104 patients who underwent surgical repair of RSOV, the early \& late mortality was $2 \%$ over a follow up of 20-year period. Yadav et al. [4], used bicameral approach for all patients in their study and concluded that the treatment of choice for RSOV aneurysm is surgical repair with an in-hospital mortality rate of $5.2 \%$ and a 5 -year survival rate of $95 \%$ and freedom from reoperation was $100 \%$. Determinants of success of repair must be patientcentric and parameters from patients based on quality of life must be considered in evaluating the success of any form of treatment. In our study, albeit there are three modalities being considered for comparison, we have extensively tried to field NYHA functional class as a prognostic variable to determine outcomes of the three treatment techniques in a larger effort to ascertain the best possible technique that is patientspecific. We strongly believe that concomitant lesions must be addressed in the same sitting as the repair without subjecting patients to adverse risks with multiple procedures, instead considered straight for surgery. RSOV cases without associated lesions and competent aortic valve function must be candidates for device closure. This helps streamline the bracket of patients with additional disease to receive non-competitive and customized treatment in lieu of exposure to unnecessary peri and post-procedural risks.

\section{Conclusion:}

Though there is a significant difference with respect to short term outcomes such as earlier improvement in NYHA functional class \& quality of life with device closure compared to surgical repair of RSOV, there is no discernable difference observed between the 2 treatment groups in terms of mortality across short term and 20 year follow up period and surgical repair is associated with less failure rate compared to device closure of RSOV and associated lesions can be addressed in the same sitting, thus we conclude that in patients with RSOV the option of device closure vs surgical repair should be chosen judiciously based on the clinical 
presentation of the patient, morphology of the RSOV and other associated lesions.

\section{References}

1. Yan F, Huo Q, Qiao J, Murat V, Ma SF. Surgery for sinus of valsalva aneurysm: 27-year experience with 100 patients. Asian Cardiovasc Thor Ann. 2008; 16(5) :361-5.

2. Post MC, Braam RL, Groenemeijer BE, Nicastia D, Rensing BJ, Schepens MA. Rupture of right coronary sinus of Valsalva aneurysm into right ventricle. Neth Heart J. 2010; 18(4) : 209-11.

3. Cheng TO, Lin ML, Gu ZQ. Aneurysm of the sinus of Valsalva: a roentgenologic study of 105 Chinese patients. Am Heart J. 1987; 114(5) :1169-77.

4. Yadav A, Mathur R, Devgarha S, Abraham V, Sisodia A. Surgery for ruptured sinus of Valsalva aneurysm: Five-year experience with 19 patients. Turk Gogus Kalp Dama. 2014 ; 22:729-33.

5. Xin-jin L, Xuan L, Bo P, Hong-wei G, Wei W, Shou-jun L, Sheng-shou H. Modified Sakakibara classification system for ruptured sinus of Valsalva aneurysm. Jour Thor Cardiovasc Surg. 2013; 146(4): $874-8$.

6. Kuriakose EM, Bhatla P, McElhinney DB. Comparison of reported outcomes with percutaneous versus surgical closure of ruptured sinus of Valsalva aneurysm. Am J Card. 2015; 115(3): 392-8.

7. Moustafa S, Mookadam F, Cooper L, Adam G, Zehr K, Stulak J, Holmes D. Sinus of Valsalva aneurysms - 47 years of a single center experience and systematic overview of published reports. Am J Card. 2007; 99(8) :1159-64.

8. Choudhary SK, Bhan A, Sharma R, Airan B, Kumar AS, Venugopal P. Sinus of Valsalva aneurysms: 20 years' experience. J Card Surg. 1997;12: 300-8.

\section{Hosted file}

Table1.pdf available at https://authorea.com/users/411035/articles/520200-outcomes-ofruptured-sinus-of-valsalva-aneurysm-a-20-year-experience

\section{Hosted file}

Table2.pdf available at https://authorea.com/users/411035/articles/520200-outcomes-ofruptured-sinus-of-valsalva-aneurysm-a-20-year-experience

\section{Hosted file}

Table3.pdf available at https://authorea.com/users/411035/articles/520200-outcomes-ofruptured-sinus-of-valsalva-aneurysm-a-20-year-experience

\section{Hosted file}

Table4.pdf available at https://authorea.com/users/411035/articles/520200-outcomes-ofruptured-sinus-of-valsalva-aneurysm-a-20-year-experience 
Freedom from Symptoms
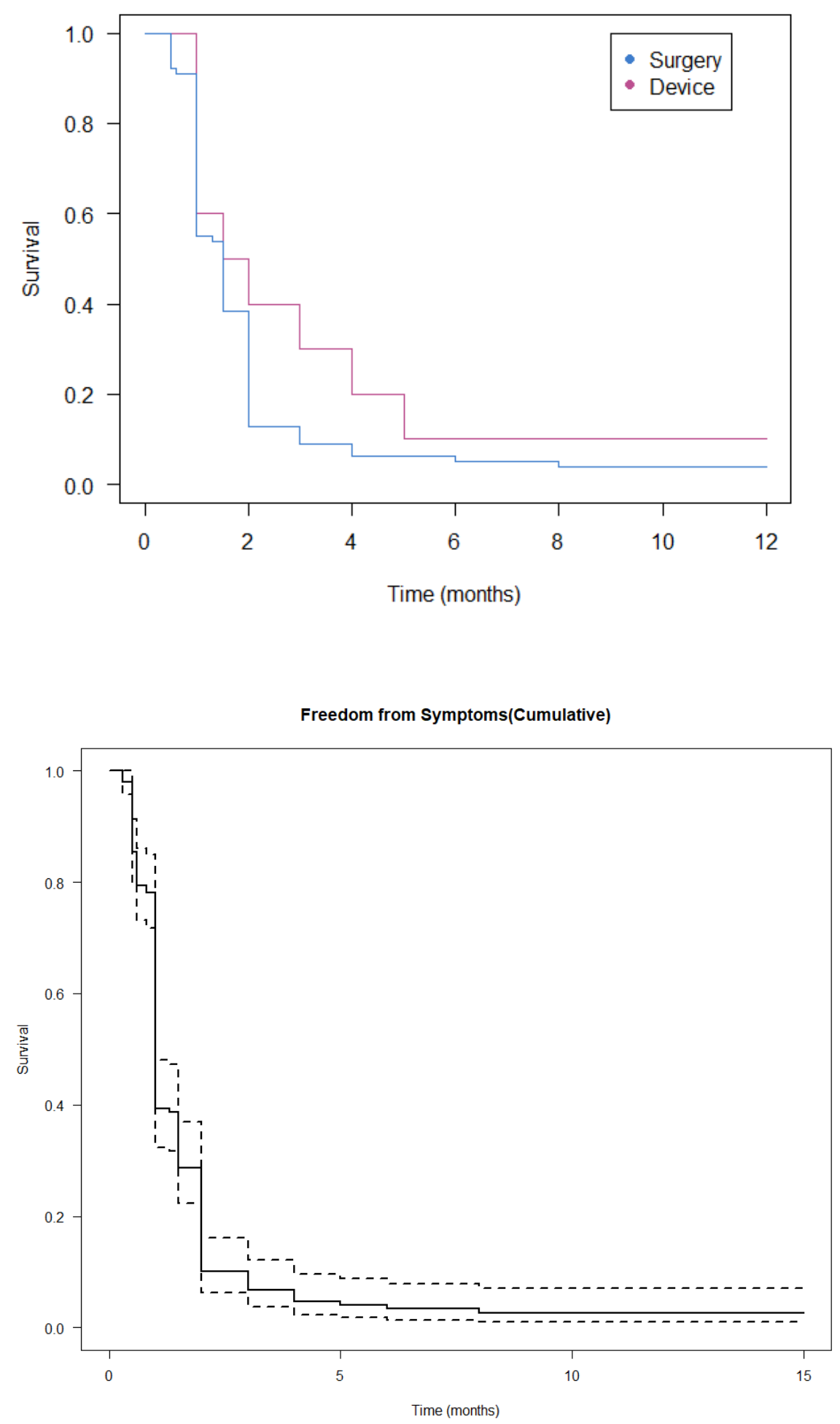


\section{Cumulative Survival following RSOV Repair}

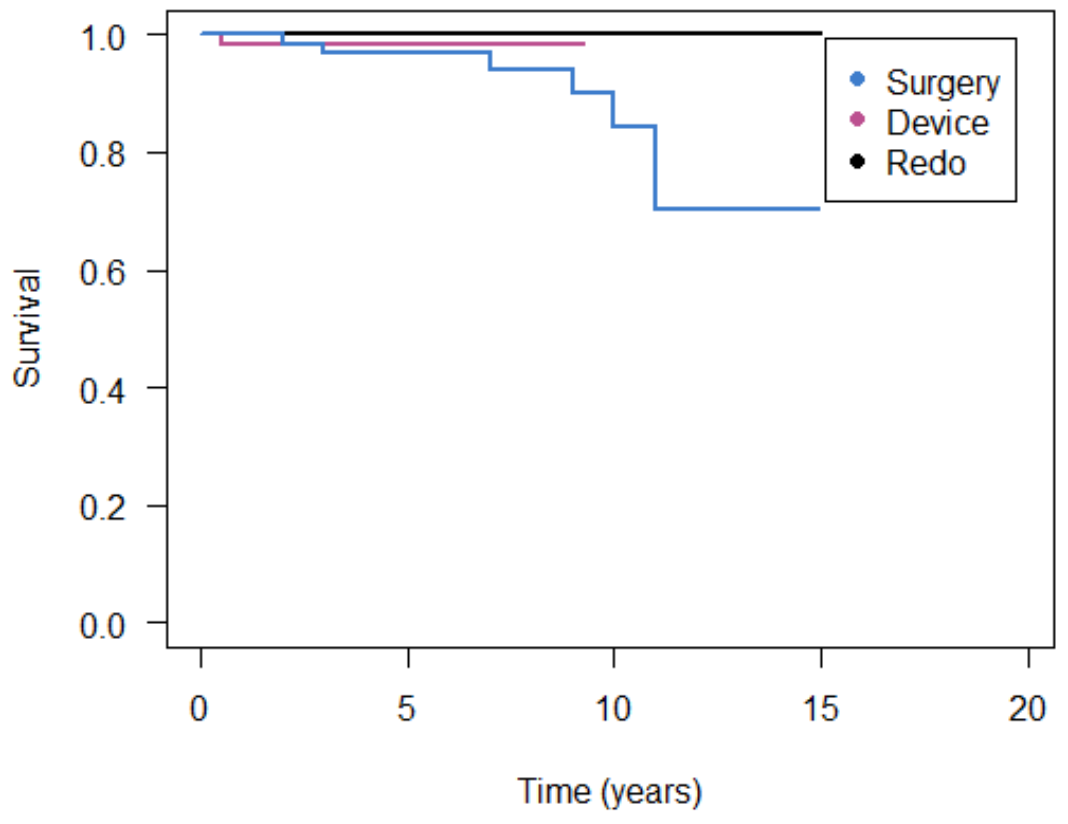

Cumulative Survival following RSOV Repair

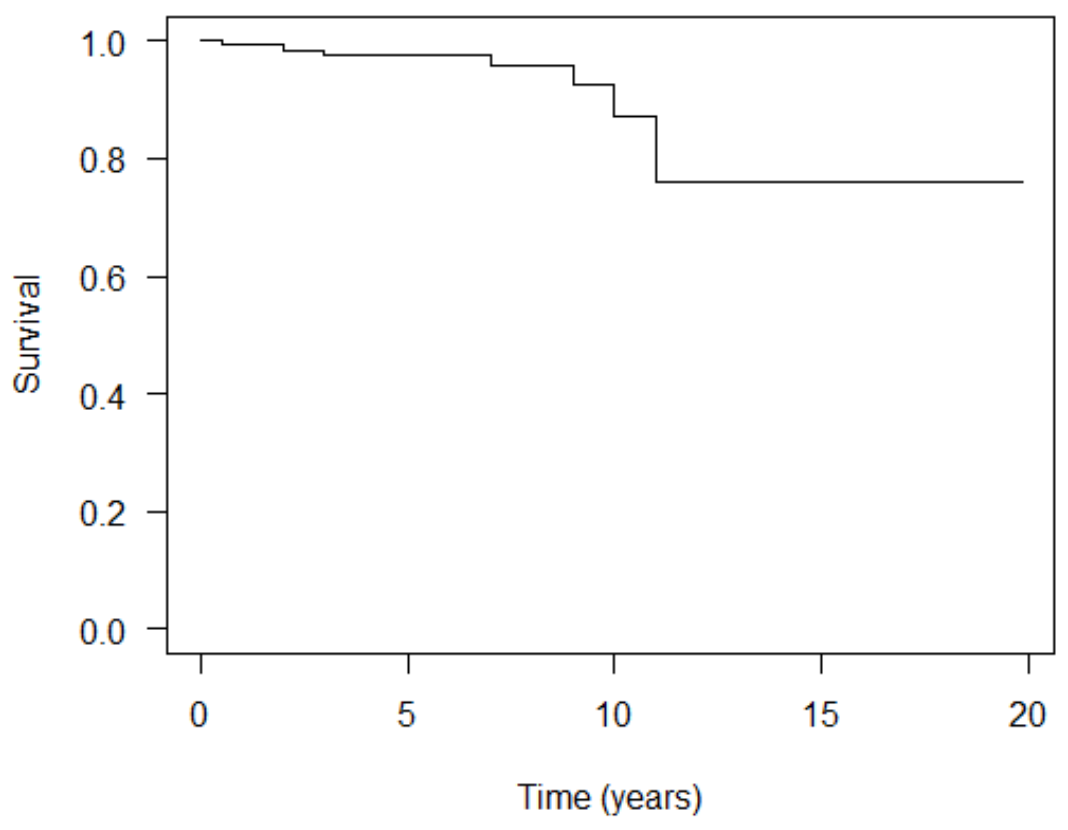

